\title{
Evaluación del subregistro de la mortalidad materna en Los Altos de Chiapas mediante las estrategias RAMOS y RAMOS modificada
}

\author{
Graciela Freyermuth-Enciso, Dra Antr Soc, (1) Rosario Cárdenas-Elizalde, Dra Est Pob y Sal Int. ${ }^{(2)}$
}

\section{Freyermuth-Enciso G, Cárdenas-Elizalde R. Evaluación del subregistro de la mortalidad materna en Los Altos de Chiapas mediante las estrategias RAMOS y RAMOS modificada. Salud Publica Mex 2009;5 I:450-457.}

\begin{abstract}
Resumen
Objetivos. Evaluar el subregistro de la mortalidad materna en Los Altos de Chiapas y reconocer la utilidad de la estrategia RAMOS modificada implementada por la Secretaría de Salud. Material y métodos. Identificación de la totalidad de las defunciones de mujeres en edad fecunda (método RAMOS) y realización de autopsias verbales. Resultados. Las autopsias verbales muestran el subregistro de 19 defunciones maternas. La estrategia RAMOS modificada que desarrolló la Secretaría de Salud sólo habría permitido identificar la ocurrencia de ocho de éstas. Conclusión. Los resultados señalan el mejoramiento de la calidad de la certificación de la causa de muerte como una prioridad para la disminución del subregistro de la mortalidad materna; de igual forma, sugieren la necesidad de evitar que los programas gubernamentales destinados a determinar la distribución de recursos financieros o de otro tipo se conviertan en posibles incentivos para la omisión de la ocurrencia de defunciones maternas.
\end{abstract}

Palabras clave: mortalidad materna; mortalidad; población indígena; registros de mortalidad; recolección de datos; México
Freyermuth-Enciso G, Cárdenas-Elizalde R. Assessment of maternal mortality underreporting in Los Altos de Chiapas using RAMOS and RAMOS modified methods. Salud Publica Mex 2009;5 1:450-457.

\begin{abstract}
Objectives.To evaluate underreporting of maternal mortality in Los Altos de Chiapas and to compare the results of this study against the RAMOS modified program conducted by the Ministry of Health. Material and Methods. A survey of all deaths of reproductive-aged women was carried out, followed by verbal autopsy interviews. Results. A total of 226 deaths of reproductive-aged women were identified and 190 verbal autopsies were carried out. Verbal autopsies showed an underreporting of 19 maternal deaths. The method used by the Ministry of Health would have led to the detection of only eight out of these 19 deaths. Conclusions. Results of this study emphasize the urgency to improve the quality of cause of death certification in order to reduce maternal mortality underreporting. They also suggest the need to prevent government programs that allocate financial resources from creating incentives for concealing maternal deaths.
\end{abstract}

Key words: maternal mortality; mortality; indigenous population; mortality registries; data collection; Mexico

(I) Centro de Investigaciones y Estudios Superiores en Antropología Social del Sureste. San Cristóbal de las Casas, Chiapas, México

(2) Universidad Autónoma Metropolitana-Xochimilco. México, DF, México.

Fecha de recibido: 15 de abril de 2009 • Fecha de aceptado: 4 de agosto de 2009 Solicitud de sobretiros: Dra. Graciela Freyermuth Enciso. Centro de Investigaciones y Estudios Superiores en Antropología Social del Sureste. Carr. San Cristóbal-San Juan Chamula, Km. 3.5, Barrio Quinta San Martín s/n. 29247 San Cristóbal de las Casas, Chiapas.

Correo electrónico: gracielafreyermuth54@hotmail.com 
A pesar de los acuerdos internacionales suscritos en encuentros como la Conferencia Internacional sobre Maternidad sin Riesgo (1987), la Cumbre a Favor de la Infancia (1990), la Conferencia Internacional sobre Población y Desarrollo (1994) o la Cuarta Conferencia Mundial sobre la Mujer (1995), la mortalidad materna es todavía uno de los problemas de salud pública más apremiantes a escala mundial. El reconocimiento de la factibilidad de disminuir la mortalidad materna ${ }^{1}$ y la importancia de obtener un compromiso político que obligue a las sociedades a lograrlo han hecho que ésta sea una de las expresiones de salud incluidas en el acuerdo denominado las Metas del Milenio. El quinto objetivo de las Metas del Milenio establece para 2015 una reducción de $75 \%$ de la mortalidad materna, en relación con la cifra reportada en 1990, en aquellos países que firmaron la iniciativa, entre ellos México.

Una de las principales dificultades que enfrenta el diseño de programas para el abatimiento de la mortalidad materna es el subregistro de las defunciones debidas a esta causa. ${ }^{2-7}$ Varios son los factores asociados al reporte incompleto del número de defunciones ocurridas por causas maternas, pero entre ellos destaca el hecho de que éstas pueden ocurrir, de acuerdo con la definición internacionalmente aceptada, no sólo durante el transcurso de la totalidad del embarazo, sino también en el parto, el posparto inmediato y los 42 días posteriores al parto (puerperio). Ello significa que las complicaciones de la gestación que tienen lugar en etapas muy tempranas del embarazo o el puerperio, las cuales pueden ocasionar el fallecimiento de la mujer, pueden no atribuirse a causas maternas. Lo anterior supone que, en países con un subregistro notorio de la mortalidad materna, el mejoramiento de la calidad del reporte de ésta requiere no sólo el reforzamiento de los sistemas de estadísticas vitales, sino también la identificación de las defunciones sospechosas de haber ocurrido como consecuencia de complicaciones del embarazo, parto o puerperio.

Una de las estrategias diseñadas para reconocer el subregistro de la mortalidad materna es la identificación de las defunciones de mujeres en edad de concebir, por lo general de 15 a 49 años, para distinguir a partir de ese punto aquéllas ocurridas por causas maternas. Esta estrategia recibe el nombre de RAMOS por sus siglas en inglés (Reproductive Age Mortality Survey) y se ha utilizado desde el decenio de 1980 en diversos países.,8-13 Si bien el expediente clínico puede proporcionar información específica que permite establecer la causa de muerte, en ocasiones no hay demanda de atención médica. Una forma de resolver la ausencia de información clínica en el caso de una defunción consiste en realizar una entrevista a familiares o personas cercanas al occiso con el propósito de establecer si su fallecimiento se debió a la causa(s) de interés. Dichas entrevistas pueden desarrollarse bajo diversas modalidades, la más frecuente de las cuales es la denominada autopsia verbal. ${ }^{14,15}$

En el año 2002, la Secretaría de Salud implementó la aplicación de la estrategia RAMOS para la identificación de defunciones maternas en México. El ejercicio propuesto por la Secretaría de Salud difirió del original en cierto grado, toda vez que en lugar de dar seguimiento a la totalidad de las defunciones de mujeres de 15 a 49 años registradas en el país para un año particular, se concentró en recabar información sobre los fallecimientos femeninos en este grupo de edad cuya causa de muerte asentada en el certificado de defunción formara parte de un conjunto de 46 causas de muerte seleccionadas. ${ }^{16}$ Esta aproximación recibió el nombre de RAMOS modificado.

Los objetivos de este trabajo son, por una parte, identificar el subregistro de la mortalidad materna en Los Altos de Chiapas y, por otra, contrastar los resultados de la aplicación de la estrategia RAMOS en la evaluación de la mortalidad materna y sus causas con los que se obtendrían al emplear la modificación propuesta por la Secretaría de Salud.

\section{Material y métodos}

Los Altos de Chiapas es la región del país con la mayor densidad de población indígena por kilómetro cuadrado. Aunque el número promedio de hablantes de lengua indígena en la región es de $80 \%$, en 14 de los 17 municipios que la conforman alcanza $95 \%$ y sólo en dos (San Cristóbal de las Casas y Teopisca) es menor de 40\%. La mayor parte de los municipios tiene una población menor de 15000 habitantes, ubicada de manera dispersa y con un acceso geográfico difícil. Para fines de este trabajo, la región de Los Altos de Chiapas corresponde a la definida por la Jurisdicción Sanitaria II del Instituto de Salud del Estado de Chiapas (ISECH) que comprende la totalidad de los municipios de la región establecida por el gobierno del Estado más el municipio de San Juan Cancuc.

El proyecto de investigación del cual deriva la información reportada en este trabajo recibió aprobación y financiamiento del Fondo Sectorial Salud-CONACyT en su convocatoria 2003. Respecto del consentimiento informado, éste se recabó de manera verbal toda vez que la mayoría de la población estudiada carece de habilidades de lectoescritura; en consecuencia, se audiograbó como parte del propio estudio.

La primera etapa del estudio comprendió la identificación de la totalidad de las defunciones femeninas de 15 a 49 años correspondientes a los municipios de interés, registradas en las oficialías del Registro Civil en 
el año 2001. En total se identificaron 226 defunciones con estas características, que conforman el universo de estudio. En la segunda etapa se contrastó la información obtenida de las oficialías del Registro Civil con la contenida en los certificados de defunción compilados por el Instituto Nacional de Geografía y Estadística (INEGI). La tercera etapa consistió en la realización de entrevistas a familiares cercanos a las mujeres fallecidas para, a través de una autopsia verbal, averiguar la causa de muerte. Los datos personales contenidos en las actas de defunción (nombres de los padres, el cónyuge y testigos y sus domicilios), así como el lugar de origen de la mujer fallecida, se emplearon como insumo para la localización de las familias por entrevistar.

Los grupos de trabajo para realizar las autopsias verbales estuvieron conformados por médicos, enfermeras, antropólogos y promotoras de salud, estas últimas bilingües con dominio del español y de al menos una de las dos lenguas indígenas habladas en la región de estudio (tzotzil y tzeltal). A fin de garantizar a los familiares estudiados un ambiente similar en términos de confidencialidad para el desarrollo de las entrevistas, las promotoras de salud no participaron en la realización de autopsias verbales en sus comunidades de origen.

La capacitación del equipo de investigación consistió, primero, en la distribución de material bibliográfico sobre mortalidad materna y la recolección de su percepción acerca de este problema de salud, los factores asociados con su ocurrencia y su importancia. Estas sesiones de trabajo permitieron al equipo compartir sus experiencias y conformar un marco común para la realización de las actividades de campo. Los ejercicios llevados a cabo estuvieron encaminados a desarrollar habilidades para la competencia cultural en los entrevistadores y los elementos para presentar de manera clara y objetiva el propósito del estudio, así como los aspectos necesarios para garantizar la participación de la población bajo un esquema de consentimiento informado.

La entrevista siguió un formato libre y su desarrollo estuvo orientado por un algoritmo organizado para la recuperación de la presencia de un embarazo al momento del fallecimiento o hasta seis semanas anteriores a la defunción, los principales signos o manifestaciones de complicaciones, su ubicación temporal en relación con el embarazo y la utilización de servicios informales y formales de salud.

Los médicos del equipo de trabajo establecieron la causa de muerte a partir de los datos obtenidos en la entrevista, que luego se compararon con los asentados en las actas y certificados de defunción. A diferencia de otros estudios en los que se emplea como criterio para señalar la causa de muerte derivada de la información recabada en entrevistas la coincidencia de opinión de al menos dos de tres médicos, en este trabajo se alcanzó un consenso acerca de la causa de muerte evidenciada por la autopsia verbal.

Las autopsias verbales se audiograbaron siempre y cuando los familiares otorgaran su consentimiento. Hasta $81.5 \%$ del total de las autopsias verbales realizadas se audiograbó. Las grabaciones en lengua indígena se utilizaron para efectuar la traducción al español y se transcribieron y audiograbaron también. A fin de garantizar la calidad de la traducción, ésta fue revisada por un hablante de la lengua indígena diferente de quien participó en la realización original de la autopsia verbal. La información relevante de cada una de las actas y certificados de defunción y de las autopsias verbales se sistematizó para su análisis y registró en una base de datos organizada con el programa Access.

La causa de muerte asentada en el certificado de defunción de las mujeres que se identificaron como perecidas por causas maternas a través de las autopsias verbales se comparó con el listado de las 46 causas seleccionadas que conforman la estrategia RAMOS modificada a fin de evaluar la eficacia de esta última.

\section{Resultados}

Se realizaron 190 autopsias verbales de las 226 defunciones de interés, lo que representa $84 \%$ del total de las defunciones femeninas de 15 a 49 años registradas en los municipios de interés en 2001. El cuadro I muestra la información relativa a las defunciones y autopsias verbales llevadas a cabo de acuerdo con los municipios. Llama la atención que dos terceras partes de los casos en los cuales no fue posible realizar la autopsia verbal se concentren en San Cristóbal de las Casas (cabecera municipal). Sin embargo, de los 24 casos ubicados en este municipio, sólo cinco no accedieron a que la autopsia verbal se llevara a cabo. De los 19 restantes, en 11 no fue posible localizar a la familia, dos correspondían a domicilios ubicados en municipios fuera de la zona de estudio y cuatro fallecimientos habían sido resultado de accidentes automovilísticos de personas que se encontraban en tránsito en la zona (cuadro II).

Las autopsias verbales permitieron identificar un subregistro de 19 defunciones por causas maternas. El cuadro III muestra la comparación entre las causas de muerte específicas asentadas en los certificados de defunción para estos 19 casos y señala cuáles recibieron atención médica y cuáles se derivaron de las autopsias verbales. Tres capítulos de causas de muerte explican más de $80 \%$ del total de los errores en el diagnóstico de las defunciones maternas encontradas a través de las autopsias verbales: enfermedades de los sistemas digestivo y respiratorio y causas no clasificadas en otro 


\section{Cuadro I}

Defunciones de mujeres de I5 a 49 años Registradas EN OFICIALÍAS Y NÚMERO DE AUTOPSIAS VERBALES REALIZADAS DE ACUERDO CON MUNCIPIOS. Los Altos de Chiapas, México, 200 I

$\begin{array}{cccc} & \begin{array}{c}\text { Defunciones } \\ \text { femeninas }\end{array} & \begin{array}{c}\text { Autopsias } \\ \text { verbales }\end{array} & \begin{array}{c}\text { Casos } \sin \\ \text { autopsia }\end{array} \\ \text { Municipio } & \text { de } 15-49 \text { años } & \text { realizadas } & \text { verbal }\end{array}$

\begin{tabular}{lrrr} 
Altamirano & 2 & 2 & 0 \\
\hline Amatenango del Valle & 2 & 2 & 0 \\
\hline Chalchihuitán & 12 & $1 \mathrm{I}$ & $\mathrm{I}$ \\
\hline Chamula & 35 & 31 & 4 \\
\hline Chanal & 3 & 3 & 0 \\
\hline Chenalhó & 15 & 15 & 0 \\
\hline Huixtán & $1 \mathrm{I}$ & $1 \mathrm{I}$ & 0 \\
\hline Larrainzar & 4 & 4 & 0 \\
\hline Mitontic & 4 & 2 & 2 \\
\hline Oxchuc & 17 & 15 & 2 \\
\hline Pantelhó & 7 & 5 & 2 \\
\hline Las Rosas & 8 & 8 & 0 \\
\hline San Cristóbal de las Casas & 55 & 31 & 24 \\
\hline Tenejapa & 21 & 21 & 0 \\
\hline Teopisca & 12 & 12 & 0 \\
\hline Zinacantán & 9 & 9 & 0 \\
\hline San Juan Cancuc & 9 & 8 & 1 \\
\hline Total & 226 & 190 & 36
\end{tabular}

Fuente: Elaboración propia con base en información recopilada en oficialías y trabajo de campo

\section{Cuadro II}

Motivos para no Realizar aUtopsias Verbales. Los Altos de Chiapas, México, 2001

Motivo

\section{San Cristóbal Otros}

de las Casas municipios Total

Familiar se negó a proporcionar información No se localizó a los familiares

Familiares no viven en la localidad

Mujer residente de otro municipio

Geográficamente inaccesible

Defunciones en accidentes automovilísticos

Casos sin suficiente información

Total

\begin{tabular}{rrr}
5 & 2 & 7 \\
4 & 2 & 6 \\
7 & 5 & 12 \\
\hline 2 & & 2 \\
\hline & 1 & 1 \\
\hline 4 & & 4 \\
\hline 2 & 2 & 4 \\
\hline 24 & 12 & 36
\end{tabular}

Fuente: Elaboración propia con base en trabajo de campo

capítulo, entre las cuales se ubican las mal definidas. La declaración consignada en el certificado de defunción de haber recibido atención médica no parece hacer ninguna diferencia respecto de los errores de diagnóstico de la causa de muerte, toda vez que tanto las causas mal definidas como las enfermedades del sistema digestivo, e incluso las particulares como neumonía inespecífica, se observaron al margen de la utilización de los servicios de salud. Cabe señalar que la autopsia verbal permitió

\section{Cuadro III \\ Comparación de CaUSAS ESPECífICAS REGISTRADAS EN LOS CERTIFICADOS DE DEFUNCIÓN Y DERIVADAS DE AUTOPSIAS VERBALES PARA 19 MUERTES MATERNAS SUBREGISTRADAS,} de acuerdo con declaración de haber recibido atención médica. Los Altos de Chiapas, México, 200 I

No. de caso Certificado de defunción*

Causa específica de defunción Autopsia verbal

Defunciones sin atención médica

\begin{tabular}{cll}
\hline I & Enfermedad arterioesclerótica del corazón (I25.I) & Hemorragia posaborto \\
\hline 2 & Otras causas mal definidas (R99) & Hemorragia posparto \\
\hline 3 & Otras causas mal definidas (R99) & Preeclampsia y desprendimiento prematuro de placenta \\
\hline 4 & Úlcera gástrica (K25.9) & Hemorragia posparto \\
\hline 5 & Perforación intestinal (K63.I) & Parto obstruido \\
\hline 6 & Enfermedad del estómago y duodeno, no especificada (K3I.9) & Muerte materna indirecta por cáncer \\
\hline 7 & Otras convulsiones y las no especificadas (R56.8) & Eclampsia \\
\hline 8 & Fiebre, no especificada (R50.9) & Sepsis puerperal \\
\hline 9 & Influenza, virus no identificado (JII.I) & Sangrado retroplacentario o rotura uterina \\
\hline I0 & Abdomen agudo (RI0.0) & Parto prolongado, o atonía o rotura uterinas \\
\hline II & Apendicitis aguda, no especificada (K35.9) & Rotura uterina posparto inmediato \\
\hline I2 & Neumonía, no especificada (JI8.9) & Sepsis puerperal \\
\hline I3 & Gastritis, no especificada (K29.7) & Eclampsia o muerte materna indirecta \\
\hline I4 & Neumonía, no especificada (J18.9) & Muerte materna posparto
\end{tabular}

Defunciones con atención médica

\begin{tabular}{lll}
15 & Anemia de tipo no especificado (D64.9) & Sepsis puerperal \\
\hline 16 & Otras obstrucciones intestinales (K56.6) & Probable rotura uterina o eclampsia \\
\hline 17 & Otras causas mal definidas (R99) & Parto obstruido \\
\hline 18 & Anemia de tipo no especificado (D64.9) & Hemorragia posparto \\
\hline 19 & Neumonía, no especificada (J18.9) & Muerte materna indirecta por tuberculosis
\end{tabular}

* Los códigos de la décima revisión de la Clasificación Internacional de Enfermedades se presentan entre paréntesis

Fuente: Elaboración propia con base en certificados de defunción (INEGI) y trabajo de campo 
detectar no sólo defunciones maternas directas, sino incluso también indirectas. Si bien, en algunos casos, el equipo no pudo determinar con precisión la causa específica de muerte a partir de la información de autopsia verbal, sí pudo distinguir de forma incuestionable que se trató de defunciones vinculadas con complicaciones del embarazo, parto o puerperio.

El cuadro IV muestra los resultados que se habrían obtenido en la búsqueda intencional de defunciones maternas subregistradas en caso de aplicar la estrategia RAMOS modificada propuesta por la Secretaría de Salud. La utilización del conjunto de 46 causas seleccionadas como elemento identificador de defunciones femeninas de 15 a 49 años, susceptibles de analizarse en profundidad, habría detectado tan sólo ocho de las 19 defunciones encontradas por este estudio al emplear la estrategia RAMOS. El capítulo de causas de muerte, en el cual la estrategia RAMOS modificada es menos sensible para la identificación de defunciones maternas subregistradas, es el de enfermedades del sistema digestivo. De las seis defunciones maternas erróneamente certificadas como debidas a alguna causa digestiva, ninguna se habría identificado mediante la estrategia RAMOS modificada propuesta por la Secretaría de Salud. De igual forma, no habría sido posible identificar el subregistro de tres defunciones incorrectamente certificadas como secundarias a otras causas mal definidas (código R99 de la $10^{\circ}$ revisión de la Clasificación Internacional de Enfermedades) toda vez que el código correspondiente no se encuentra incluido en la lista de 46 causas que conforma el programa RAMOS modificado (cuadro V).

\section{Discusión}

Este estudio pone de manifiesto la persistencia de los errores de clasificación en la causa de muerte como uno de los componentes del subregistro de la mortalidad materna en la región Los Altos de Chiapas, así como las limitaciones del método RAMOS modificado implementado por la Secretaría de Salud para la identificación de defunciones maternas subregistradas. Sin embargo, cabe recordar que la zona del país en la cual se realizó este estudio es una de las de mayor mortalidad materna y con rezagos sociodemográficos y de acceso a servicios. Los diferenciales observados en el país obligan a ser cautelosos al pretender generalizar los resultados reportados en este estudio a otras regiones. El trabajo consignado en este estudio requirió el seguimiento de 190 defunciones de mujeres en edad fecunda para identificar 19 defunciones maternas. El método RAMOS modificado hubiera partido de un número de defunciones menor, 59 casos, dado que sólo habría incluido las defunciones de mujeres en edad de procrear cuya causa de fallecimiento correspondiera a alguna de las 46 causas que conforman la lista, con lo cual se habría identificado, de acuerdo con estos resultados, un máximo de ocho defunciones maternas. Pese a que en primera instancia la ampliación de la lista de causas comprendidas en el método RAMOS modificado puede presentarse como una alternativa ante las deficiencias en la identificación de muertes maternas mal clasificadas, incluso hasta llegar a adoptarse el RAMOS original, los esfuerzos para el mejoramiento del registro de la mor-

Cuadro IV

Comparación de los RESUltados obTENIDOS CON RAMOS Y RAMOS MOdificado en la identificación de 19 muertes maternas. Los Altos de Chiapas, México, 200 I

\begin{tabular}{|c|c|c|c|c|}
\hline Capítulo de causa de muerte & $\begin{array}{l}\text { Códigos de causa de muerte } 10^{a} \text { Revisión de } \\
\qquad \text { la CIE* }\end{array}$ & $\begin{array}{l}\text { Identificadas } \\
\text { con RAMOS }\end{array}$ & $\begin{array}{c}\text { Identificables } \\
\text { con RAMOS } \\
\text { modificado }\end{array}$ & $\begin{array}{l}\text { No identifi- } \\
\text { cables con } \\
\text { RAMOS } \\
\text { modificado }\end{array}$ \\
\hline Enfermedades de la sangre y los órganos hematopoyéticos & D64.9, D64.9 & 2 & 2 & \\
\hline Enfermedades del sistema circulatorio & 125.1 & I & & I \\
\hline Enfermedades del sistema respiratorio & $\mathrm{JII.I,JI8.9,J|8.9,J18.9}$ & 4 & 3 & I \\
\hline Enfermedades del sistema digestivo & K25.9, K29.7, K31.9, K35.9, K56.6, K63.I & 6 & & 6 \\
\hline $\begin{array}{l}\text { Síntomas, signos y hallazgos anormales clínicos y de labora- } \\
\text { torio no clasificados en otra parte }\end{array}$ & RI0.0, R50.9, R56.8, R99, R99, R99 & 6 & 3 & 3 \\
\hline Total & & 19 & 8 & II \\
\hline
\end{tabular}

Fuente: Elaboración propia con base en trabajo de campo 


\section{CuadroV \\ Capítulos y códigos de las 46 causas de muerte que conforman la estrategia RAMOS modificada de la Secretaría de Salud, México}

\begin{tabular}{|c|c|c|}
\hline Capítulo de causa & $\begin{array}{l}\text { Códigos de la décima revisión de la Clasificación Internacional } \\
\text { de Enfermedades }\end{array}$ & $\begin{array}{l}\text { Número de } \\
\text { causas en el } \\
\text { capítulo }\end{array}$ \\
\hline Ciertas enfermedades infecciosas y parasitarias & A40,A4I & 2 \\
\hline Enfermedades de la sangre y los órganos hematopoyéticos & $\begin{array}{l}\text { D50, D62, D64.9, D65, D69.3, D69.4, D69.5, D69.6, D69.8, } \\
\text { D69.9 }\end{array}$ & 10 \\
\hline Trastornos metabólicos & E86, E87, E88.9, E89.6, E89.9 & 5 \\
\hline Enfermedades del sistema nervioso & G93.I, G93.2, G93.5, G93.6, G93.9 & 5 \\
\hline Enfermedades del sistema circulatorio & $\begin{array}{l}\text { II0-II5, I26, I46.9, I47, I48, I49, I50, I60-169 (excepto I67.I, I67.3, } \\
167.5,168.0,168.1 \text {, I68.2), I74.9 }\end{array}$ & 3 , \\
\hline Enfermedades del sistema respiratorio & $J 18, j 96$ & 2 \\
\hline Enfermedades del sistema digestivo & $\mathrm{K} 65, \mathrm{~K} 72$ & 2 \\
\hline Enfermedades del sistema genitourinario & NI7, NI9, N70-N73, N74.8, N92, N93.8, N93.9 & 4 \\
\hline $\begin{array}{l}\text { Síntomas, signos y hallazgos anormales clínicos y de laboratorio } \\
\text { no clasificados en otra parte }\end{array}$ & RI0.0, R40.2, R50, R56, R57.I, R58 & 6 \\
\hline Complicaciones de la atención médica y quirúrgica & Y48.0-Y48.4 & 1 \\
\hline Total & & 46 \\
\hline
\end{tabular}

Fuente: Referencia 16

talidad materna deben centrarse en la corrección de las deficiencias de los sistemas de registro de defunciones con los que cuenta el país. En todo caso, los resultados obtenidos delinean las defunciones debidas a causas digestivas y aquéllas certificadas como secundarias a otras causas mal definidas, como las categorías en las cuales el programa RAMOS modificado mostraría las mayores deficiencias en la identificación de causas maternas en esta zona del país; es por ello que su inclusión en dicha lista pudiera contribuir a mejorar la eficiencia de esta metodología.

En el caso concreto de los errores en la clasificación de la mortalidad materna, la actividad más importante para el mejoramiento de las fuentes de información debe focalizarse en la certificación correcta de la causa de muerte de mujeres en edad fecunda. Alrededor de 90\% de las defunciones maternas lo certifica personal de salud y apenas en una cuarta parte de éstas el certificado de defunción lo extiende el médico tratante. De lo anterior se infiere que si bien los certificados de defunción son llenados por el personal que se considera en mejores condiciones de establecer la causa de muerte, es decir, personal médico, los encargados de emitir la mayoría de éstos no necesariamente están familiarizados con el padecimiento que condujo al fallecimiento de la mujer.
Las discrepancias encontradas por este estudio entre las causas de muerte asentadas en los certificados de defunción y las obtenidas mediante las autopsias verbales son sugerentes de deficiencias clínicas por parte del personal de salud para el reconocimiento de las afecciones obstétricas que produjeron el fallecimiento o acciones deliberadas para enmascarar la ocurrencia de muertes maternas. La lógica que subyace a la implementación del RAMOS modificado responde a una situación similar a la evidenciada por este estudio (errores en la clasificación de la causa de muerte), puesto que la intención del programa es identificar, entre las defunciones de mujeres en edad de concebir certificadas como debidas a alguna de las 46 causas seleccionadas, aquellas que en realidad correspondieron a causas maternas. Esto significa que la propia Secretaría de Salud reconoce que un componente del subregistro de la mortalidad materna en el país corresponde a los errores en el establecimiento de la causa de muerte.

Un aspecto adicional a considerar en la problemática de las deficiencias en la certificación de las muertes maternas es el posible impacto de los programas sociales o de salud que condicionan la entrega de recursos financieros sobre la reducción inmediata, o en el muy corto plazo, de la mortalidad materna o incluso sobre 
la eliminación de ésta, lo que de facto podría traducirse en un incentivo para subreportar la ocurrencia de defunciones asociadas a complicaciones obstétricas.

La mejoría del registro de la mortalidad materna es una piedra angular para el diseño y evaluación de acciones destinadas a disminuir la ocurrencia de ésta. Los esfuerzos requeridos para la aplicación de la estrategia de identificación de las defunciones maternas a partir de los fallecimientos ocurridos en población en edad fecunda podrían aplicarse para instituir una evaluación de la calidad del llenado del certificado de defunción, sobre todo en relación con la causa que provocó el fallecimiento, así como al establecimiento de aquellos factores que obstaculizan la utilización oportuna de servicios de salud reproductiva, en especial los de índole obstétrica. Para el cumplimiento de estos objetivos puede establecerse un programa de autopsias a defunciones hospitalarias de mujeres en edad de procrear seleccionadas de manera aleatoria, ${ }^{17}$ así como la realización de autopsias verbales a partir de muestras aleatorias de certificados de defunción de mujeres en edad de concebir. En el caso de las autopsias a defunciones hospitalarias, la selección aleatoria propiciaría un mejoramiento de la certificación de la causa de muerte por parte del personal de salud que interviene en el otorgamiento de servicios clínicos, mientras que el de autopsias verbales permitiría acceder a información acerca de defunciones que suceden en sitios distintos de las unidades médicas. La información derivada de ambas intervenciones haría posible evaluar la calidad de la certificación médica de causa de muerte y la magnitud de los errores de clasificación de ésta. Una estrategia adicional para identificar el subregistro de la mortalidad materna consiste en el empleo combinado de la información proveniente de certificados de defunción y aquella contenida en censos de población, registros sobre nacimientos, unidades médicas o padrones de programas sociales. ${ }^{18,19}$

En zonas del país como la analizada en este estudio, los aspectos culturales desempeñan un papel crucial en la efectividad de las acciones emprendidas para reducir la mortalidad materna. La ausencia o limitada presencia de personal de salud con entrenamiento intercultural constituyen otro tipo de barrera para la atención obstétrica en regiones donde reside población indígena. Los problemas de comunicación entre la población hablante de lengua indígena y el personal de salud que no la habla pueden explicar, al menos en parte, que en los casos en los cuales la autopsia verbal puso de manifiesto el antecedente de un embarazo reciente al momento del fallecimiento, el personal de salud pudo quizá encontrar evidencia de infección (véanse los casos 8 y 12, cuadro III) pero no identificar el cuadro de sepsis puerperal.
Por último, es indispensable que el diseño e implementación de las acciones ideadas para mejorar la calidad de los registros sobre causas de muerte no se conviertan en incentivos para el ocultamiento deliberado de anomalías específicas. En este sentido, resulta imperioso garantizar que las evaluaciones de la información sobre certificación de causa de muerte se lleven a cabo en un entorno libre de prejuicio hacia las instituciones o el personal de salud y que los resultados emanados de éstas no se traduzcan en una reducción de recursos o afectación a la cobertura de los programas operativos.

\section{Referencias}

I. Högberg U,Wall S, Broström G. The impact of early medical technology on maternal mortality in late 19th century Sweden. Int J Gynaecol Obstet 1986;24:25I-26I.

2. Gissler M, Deneux-Tharaux C,Alexander S, Berg CJ, Bouvier-Colle MH, Harper M, et al. Pregnancy-related deaths in four regions of Europe and the United States in 1999-2000: Characterisation of unreported deaths. Eur J Obstet Gynecol Reprod Biol 2007; I33(2): I79-185.

3. Grubb GS, Fortney JA, Saleh S, Gadalla S, el-Baz A, Feldblum P, et al. A comparison of two cause-of-death classification systems for deaths among women of reproductive age in Menoufia, Egypt. Int J Epidemiol 1988; I7(2):385-39|.

4. Horon IL. Underreporting of maternal deaths on death certificates and the magnitude of the problem of maternal mortality.Am J Public Health 2005;95(3):478-482.

5. Kao S, Chen LM, Shi L, Weinrich MC. Underreporting and misclassification of maternal mortality in Taiwan. Acta Obstet Gynecol Scand 1997;76(7):629-636.

6. Karimian-Teherani D, Haidinger G,Waldhoer T, Beck A,Vutuc C. Underreporting of direct and indirect obstetrical deaths in Austria, 1980-98. Acta Obstet Gynecol Scand 2002;8I (4):323-327.

7. Rodríguez-Angulo E,Andueza-Pech G, Montero-Cervantes L, HoilSantos J. Subregistro de muertes materna en Mérida,Yucatán, México (1997-200I). Ginecol Obstet Mex 2005;73(7):347-354.

8. Alves SV. Maternal mortality in Pernambuco, Brazil: what has changed in ten years? Reprod Health Matters 2007; 15(30): I34- I44.

9. el Kady AA, Saleh S, Gadalla S, Fortney J, Bayoumi H. Obstetric deaths in Menoufia Governorate, Egypt. Br J Obstet Gynaecol 1989;96(I):9-14. I0. Fortney JA, Susanti I, Gadalla S, Saleh S, Rogers SM, Potts M. Reproductive mortality in two developing countries. Am J Public Health 1986;76:134-138.

I I. Mungra A, van Bokhoven SC, Florie J, van Kanten RW, van Roosmalen J, Kanhai HH. Reproductive age mortality survey to study under-reporting of maternal mortality in Surinam. Eur J Obstet Gynecol Reprod Biol 1998;77(I):37-39.

12. Türkyilmaz AS, Koc I, Schumacher R, Campbell OM. The Turkey national maternal mortality study. Eur J Contracept Reprod Health Care 2009; I4(I):75-82.

I3.Yadav H. Study of maternal deaths in Kerian (1976-1980). Med J Malaysia 1982;37(2):I65-I69.

14. Bartlett L, Mawji AS, Whitehead S, Crouse C, Dalil S, lonete D, et al. Where giving birth is a forecast of death: maternal mortality in four districts of Afghanistan, 1999-2002. Lancet 2005;365(9462):864-870. 
15. Walraven G, Telfer M, Rowley J, Ronsmans C. Maternal mortality in rural Gambia: levels, causes and contributing factors. Bull World Health Organ 2000;78(5):603-613.

16. Secretaría de Salud. Medición de la mala Clasificación de la Mortalidad Materna en México, 2002-2004, Síntesis Ejecutiva 16. México: Subsecretaría de Innovación y Calidad, Dirección General de Información en Salud, Secretaría de Salud, 2005.

17. Cárdenas R. Información estadística en salud: avances y necesidades. En: Figueroa B, coord. El dato en cuestión. Un análisis de las cifras sociodemográficas. Ciudad de México: El Colegio de México, 2008:57I-612.
18. Cliffe S, Black D, Bryant J, Sullivan E. Maternal deaths in New South Wales,Australia: a data linkage project.Aust N Z J Obstet Gynaecol 2008;48(3):255-260.

19. Gissler M, Berg C, Bouvier-Colle MH, Buekens P. Methods for identifying pregnancy-associated deaths: population-based data from Finland. Paediatr Perinat Epidemiol 2004;18(6):448-455. 